\title{
Searching for SUSY at ATLAS with \\ Trilepton and Missing Transverse Momentum
}

SUSY08, Seoull

19/06/2008
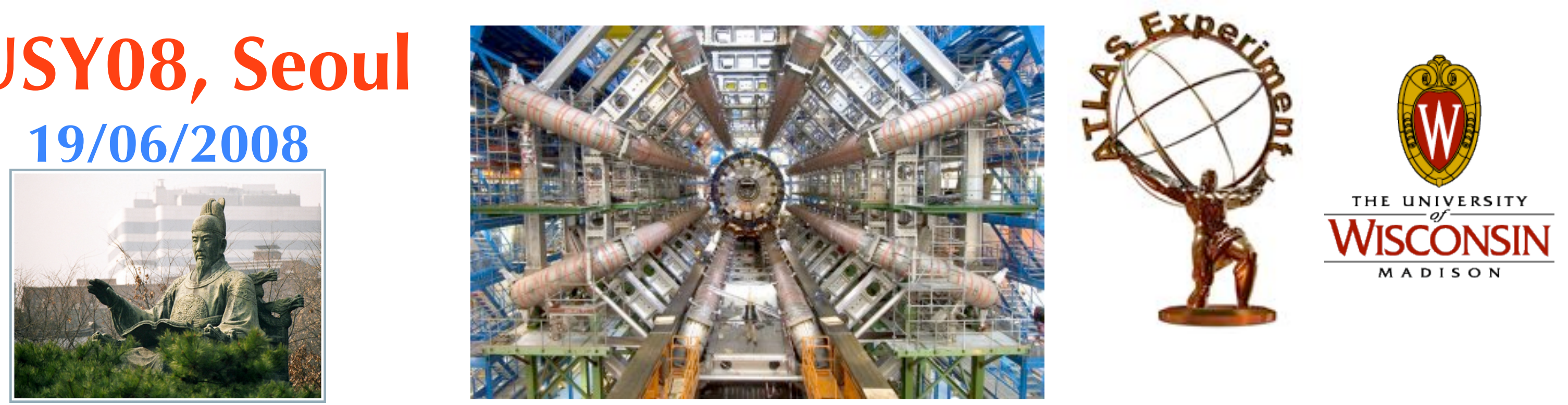

* Importance of trilepton signature * ATLAS' approach so far * Discovery potential and plans for future 


\section{Introduction}

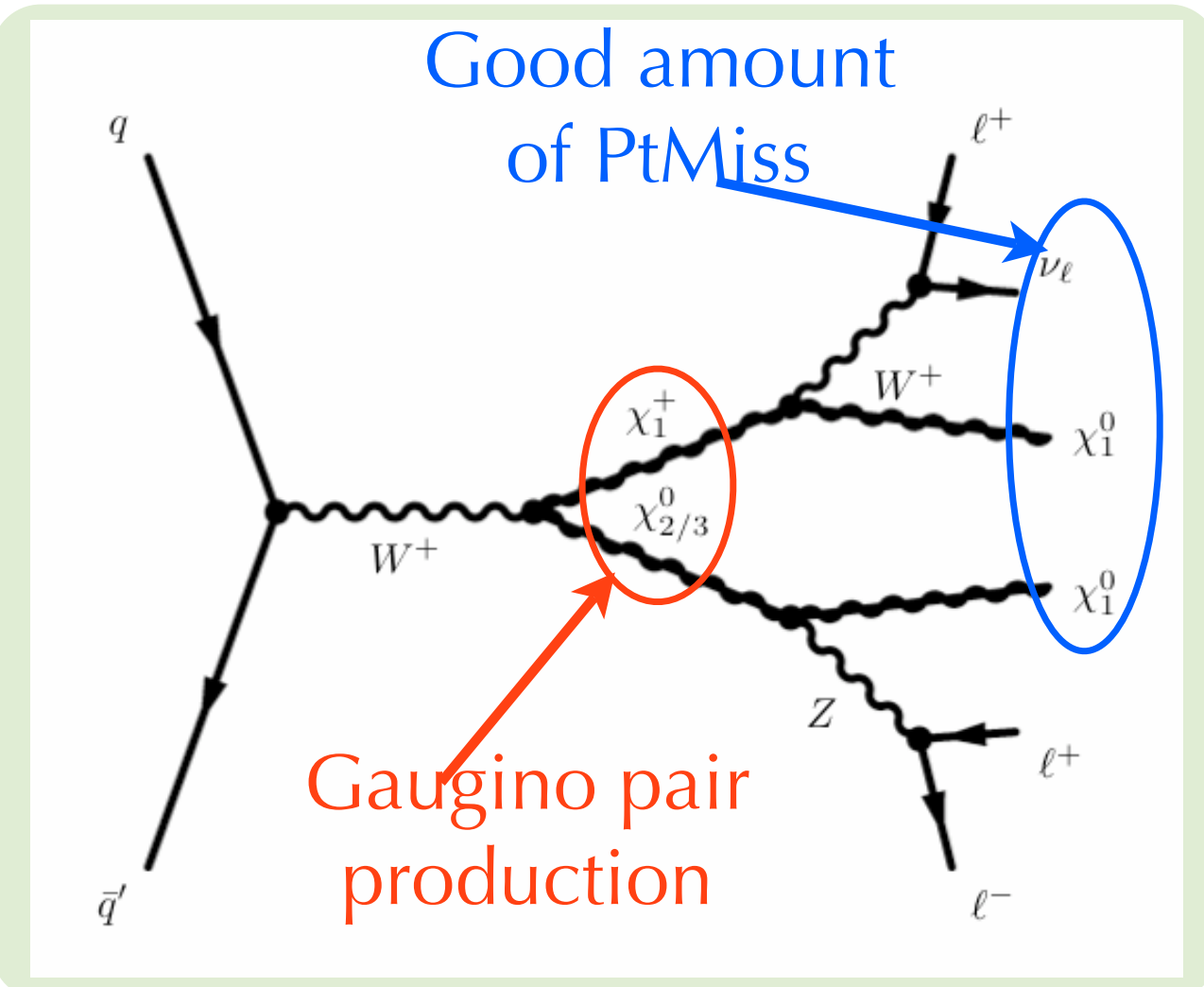

$\checkmark$ Channels with multi-lepton and missing transverse momentum (PtMiss) has always been in the SUSY discovery pool at LHC $\checkmark$ Here, we measure the discovery potential of three lepton and PtMiss with little jet activity in the final state $\checkmark$ In SUSY R-parity conserved scenario leptonic final states are produced from the pairs of heavy gaugino decay through virtual $W / Z$ decay or through sleptons

$\checkmark$ The analysis considered only the mSUGRA framework which is characterized by five parameters $\checkmark$ In ATLAS, several benchmark points are studied within these parameter space

\begin{tabular}{|c|r|r|r|r|r|}
\hline Parameter & SU1 & SU2 & SU3 & SU4 & SU8 \\
\hline \hline$m_{0}$ & 70 & 3550 & 100 & 200 & 210 \\
$m_{1 / 2}$ & 350 & 300 & 300 & 160 & 360 \\
$A_{0}$ & 0 & 0 & -300 & -400 & 0 \\
$\tan \beta$ & 10 & 10 & 6 & 10 & 40 \\
$\operatorname{sign} \mu$ & + & + & + & + & + \\
\hline
\end{tabular}




\section{Benchmark point}

$\checkmark$ For the trilepton analysis we considered $\mathrm{SU} 2$ as the important benchmark point $\checkmark$ It lies within the focus point region of $\mathrm{m}_{0}, \mathrm{~m}_{1 / 2}$ plane

$\checkmark$ This is the only region of mSUGRA parameter space where there is a larger cross-section for direct gaugino pair production than any other considered points
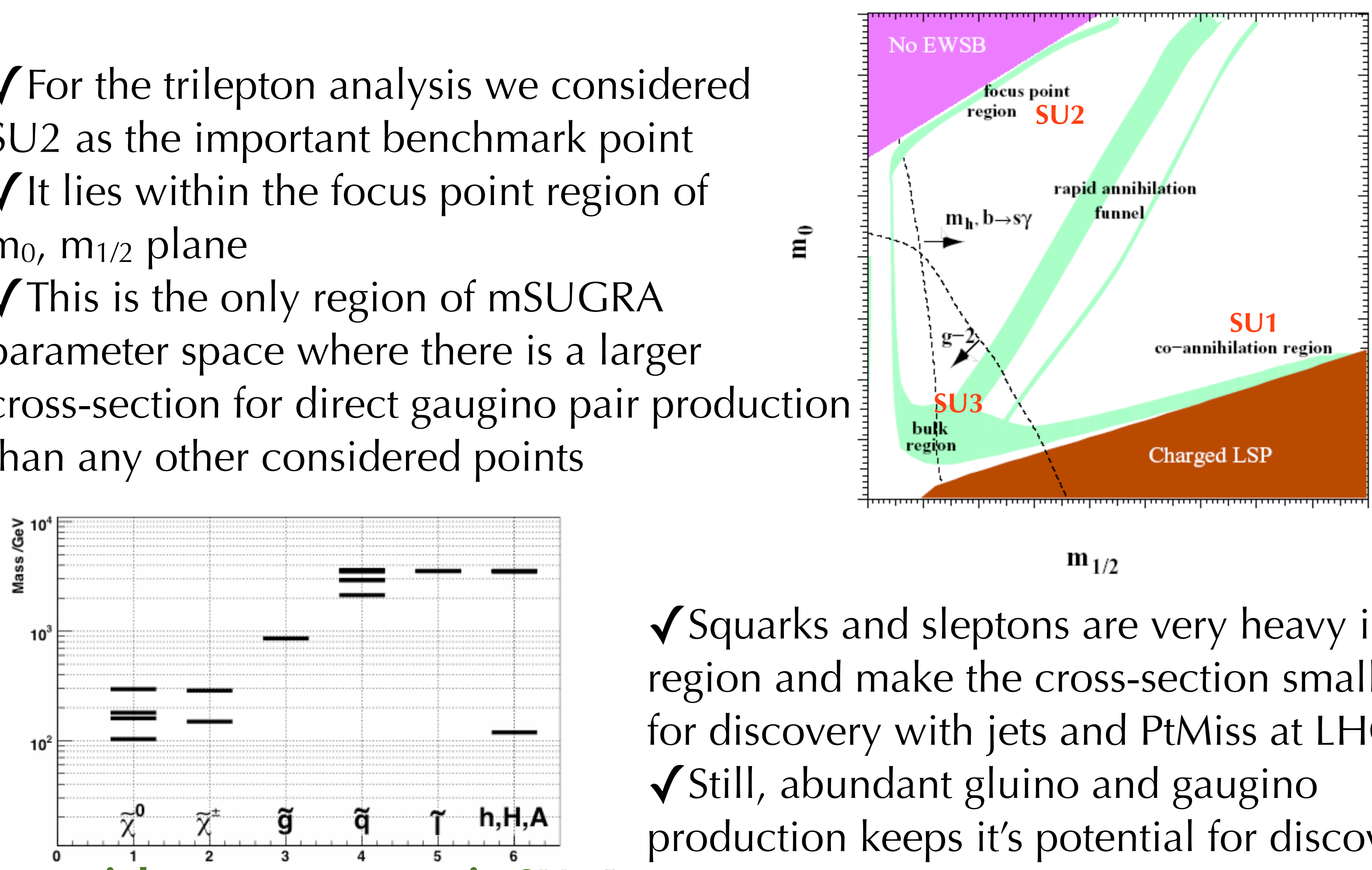

$\mathbf{m}_{1 / 2}$

$\checkmark$ Squarks and sleptons are very heavy in this region and make the cross-section smaller for discovery with jets and PtMiss at LHC $\checkmark$ Still, abundant gluino and gaugino production keeps it's potential for discovery

[ sparticle mass spectrum in SU2 ] in multi-lepton final state 


\section{Gaugino pair production in benchmark point SU2}

$\checkmark$ Exclusive trilepton signal for SU2 is dominated by the pair production of lighter chargino $\left(\tilde{\chi}_{1}^{ \pm}\right)$and $2^{\text {nd }}$ lightest neutralino $\left(\tilde{\chi}_{2}^{0}\right)$

Where, $\tilde{\chi}_{1}^{ \pm} \rightarrow \tilde{\chi}^{0} l^{ \pm} \nu$

$$
\tilde{\chi}_{2}^{0} \rightarrow \tilde{\chi}^{0} l^{+} l^{-}
$$

\begin{tabular}{|c|c|r|}
\hline Sparticle & Decay Mode & B.R. \\
\hline \hline$\tilde{\chi}_{2}^{0}$ & $\tilde{\chi}_{1}^{0} \ell^{+} \ell^{-}$ & $7 \%$ \\
\hline$\tilde{\chi}_{3}^{0}$ & $\tilde{\chi}_{1}^{0} \ell^{+} \ell^{-}$ & $7 \%$ \\
\hline$\tilde{\chi}_{4}^{0}$ & $\tilde{\chi}_{1}^{ \pm} W^{\mp}$ & $81 \%$ \\
& $\tilde{\chi}_{3}^{0} Z$ & $12 \%$ \\
\hline$\tilde{\chi}_{1}^{ \pm}$ & $\tilde{\chi}_{1}^{0} l^{ \pm} v$ & $22 \%$ \\
\hline$\tilde{\chi}_{2}^{ \pm}$ & $\tilde{\chi}_{2}^{0} W^{ \pm}$ & $38 \%$ \\
& $\tilde{\chi}_{3}^{0} W^{ \pm}$ & $18 \%$ \\
& $\tilde{\chi}_{1}^{ \pm} Z$ & $30 \%$ \\
\hline
\end{tabular}

\begin{tabular}{|c|r|r|}
\hline Production & \multicolumn{1}{|c|}{$\sigma[\mathrm{fb}]$} & Trilepton events $/ 10 \mathrm{fb}^{-1}$ \\
\hline \hline$\tilde{\chi}_{1}^{ \pm} \tilde{\chi}_{2}^{0}$ & 1138.0 & 175 \\
\hline$\tilde{\chi}_{1}^{ \pm} \tilde{\chi}_{3}^{0}$ & 679.3 & 105 \\
\hline$\tilde{\chi}_{1}^{ \pm} \tilde{\chi}_{4}^{0}$ & 51.4 & 6 \\
\hline$\tilde{\chi}_{2}^{ \pm} \tilde{\chi}_{2}^{0}$ & 58.5 & 7 \\
\hline$\tilde{\chi}_{2}^{ \pm} \tilde{\chi}_{3}^{0}$ & 61.6 & 7 \\
\hline$\tilde{\chi}_{2}^{ \pm} \tilde{\chi}_{4}^{0}$ & 310.3 & 26 \\
\hline \hline TOTAL & & 326 \\
\hline
\end{tabular}

[ Leading Order cross-sections are mentioned here ] 


\section{Lepton ID, Efficiency}

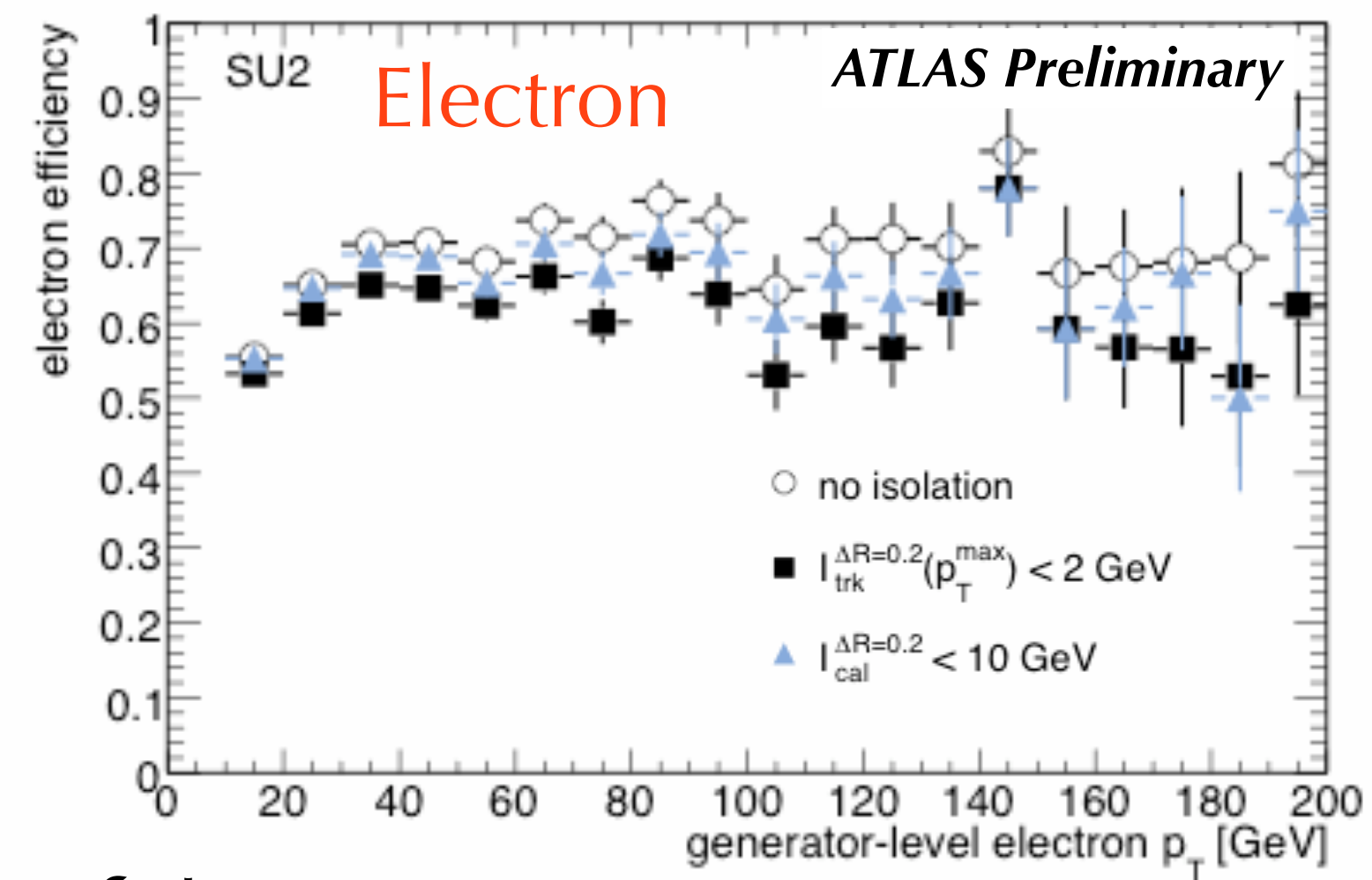

$\checkmark$ Good lepton selection criteria is crucial for measurements like this

$\checkmark$ Electron and Muon identification are done based on official ATLAS criteria $\checkmark$ In this analysis a lower Pt threshold of $10 \mathrm{GeV}$ is used in an attempt to improve the trilepton event rate

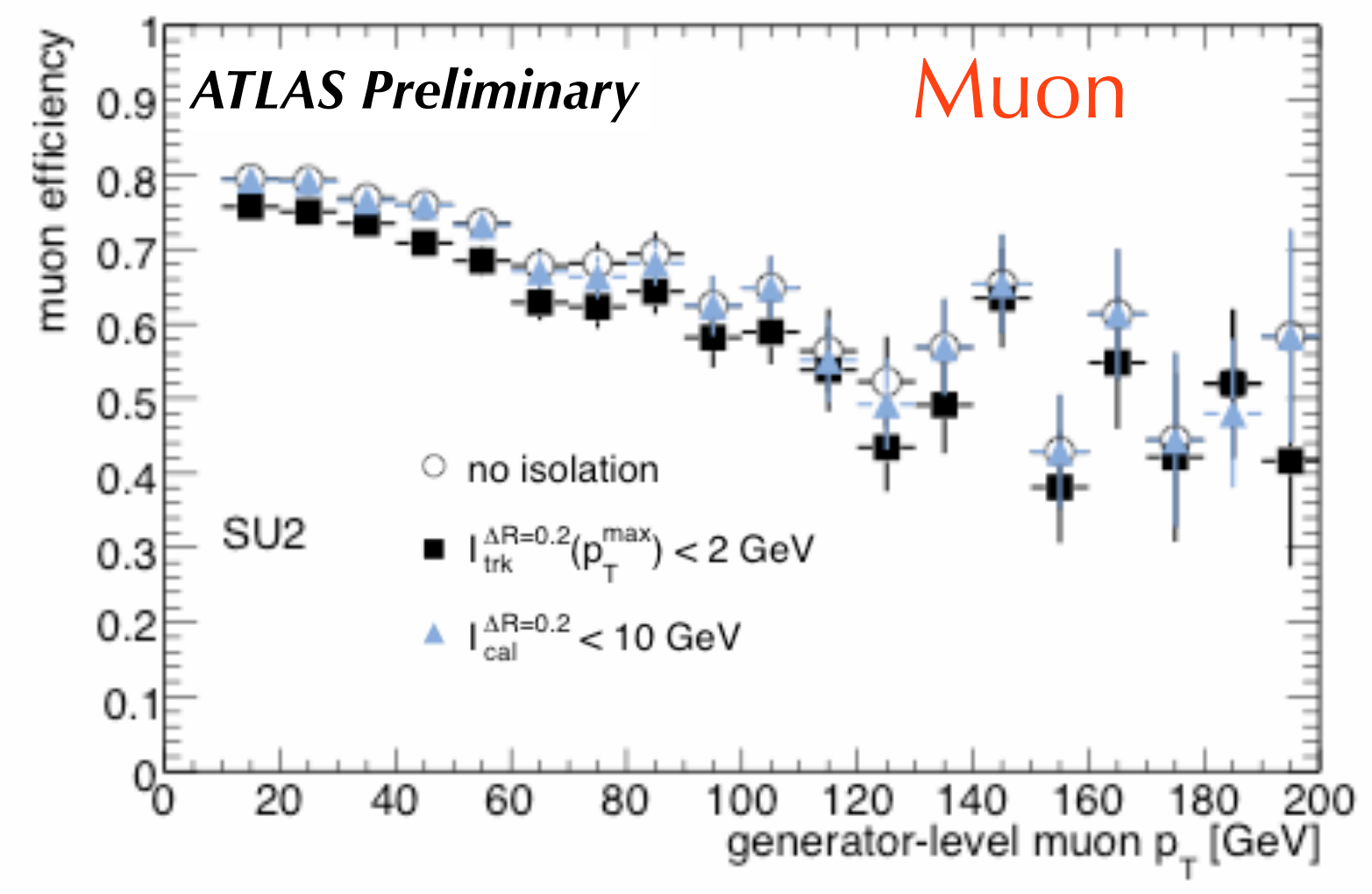

- An isolation energy in the calorimeter surrounding muon within a cone of $\mathrm{DR}<0.2$ is applied to be $<10 \mathrm{GeV}$ 


\section{Fake Lepton Rate \\ $\checkmark$ Jets :}

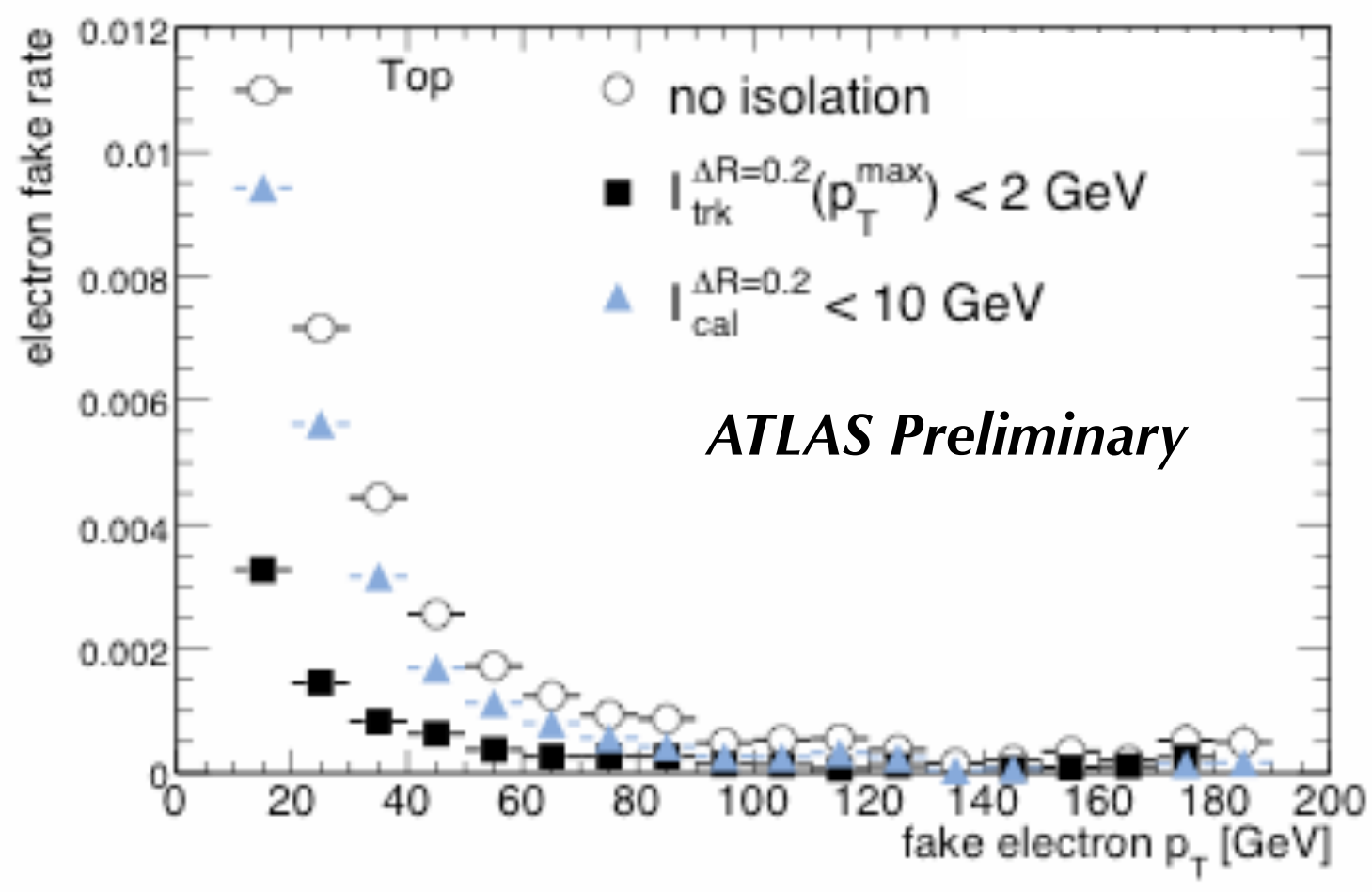

- Pt $>10 \mathrm{GeV}$, $\mid$ eta $\mid<2.5$

- Reconstructed based on calorimeter tower signals with a seeded cone algorithm of size 0.4

- Those overlap with an electron/muon within a DR(lep,jet $)<0.2$ are not considered

$\checkmark$ Veto Electrons and Muons if DR(lep, jet) $<0.4$ ( referred as no isolation in the plots )

$\checkmark$ This reduces the efficiency (few \%) but acts as very good discriminant already in reducing the fake background from top sample $\checkmark$ Using the overlap removal criteria fake rate $=4.1 \pm 0.1 \times 10-3$ (electrons)

$$
=1.1 \pm 0.1 \times 10-3 \text { (muons) }
$$

$\checkmark$ The isolation criteria provides relative suppression of fake rate : $20 \%$ (calorimeter isolation $<10 \mathrm{GeV}$ ) $74 \%$ (track isolation $<2 / 1 \mathrm{GeV}$, e/mu) 


\section{Event Selection-i}

\section{$\sqrt{ }$ Signal events are divided into two categories}

- Inclusive SUSY particle pair production

- A general case where we obtain signal from different benchmark points

- Here, the three leptons are produced in the cascade decays of heavier sparticles, typically squarks and gluinos

- Direct production of chargino and neutralino only

- This is for harder case at LHC where the final state is obtained from gaugino pair production and the coloured sparticles are very heavy to be contained with high jet activity - This scenario is sensitive to SUSY while the other signatures (requiring jets) will have more difficulties making a discovery

\section{$\checkmark$ After a 3 lepton requirement,} ttbar and $\mathrm{Zb}$ : dominant background in low Pt

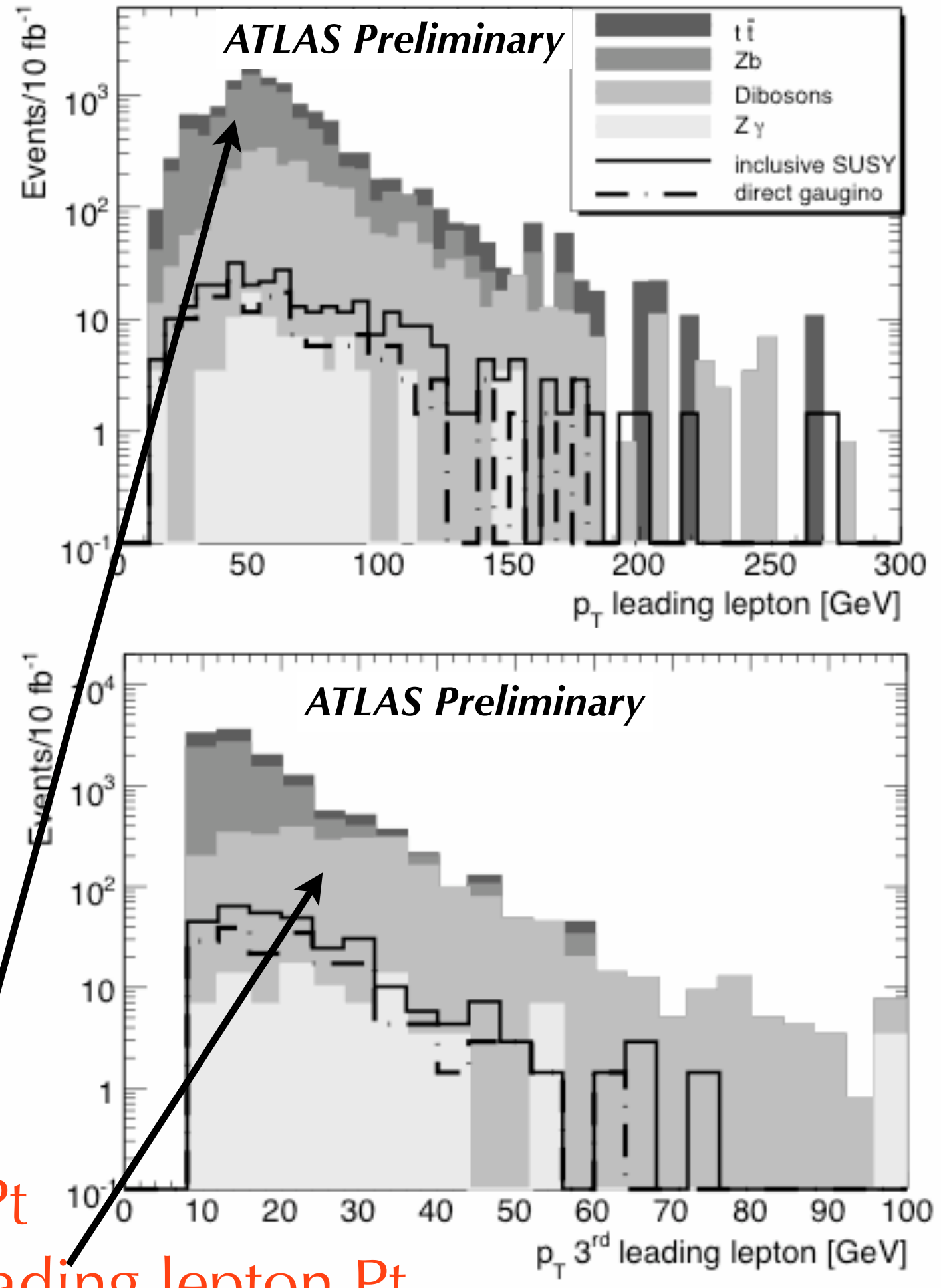
Diboson : in the entire phase space of 3 rd leading lepton Pt 


\section{Event Selection-ii}

$\checkmark$ Use more stringent track isolation cut on the leptons $\mathrm{P}_{T(t r k, \max )}^{\Delta R=0.2}$ : Maximum Pt of the tracks inside a cone of $\mathrm{DR}<0.2$ is applied to be $<1 \mathrm{GeV}$

$\checkmark$ The cut reduces $23 \%(\mathrm{tt}), 37 \%(\mathrm{Zb})$ background events. Keep 82\% (incl. SUSY), $86 \%$ (direct gaugino) signal events for SU2 benchmark point
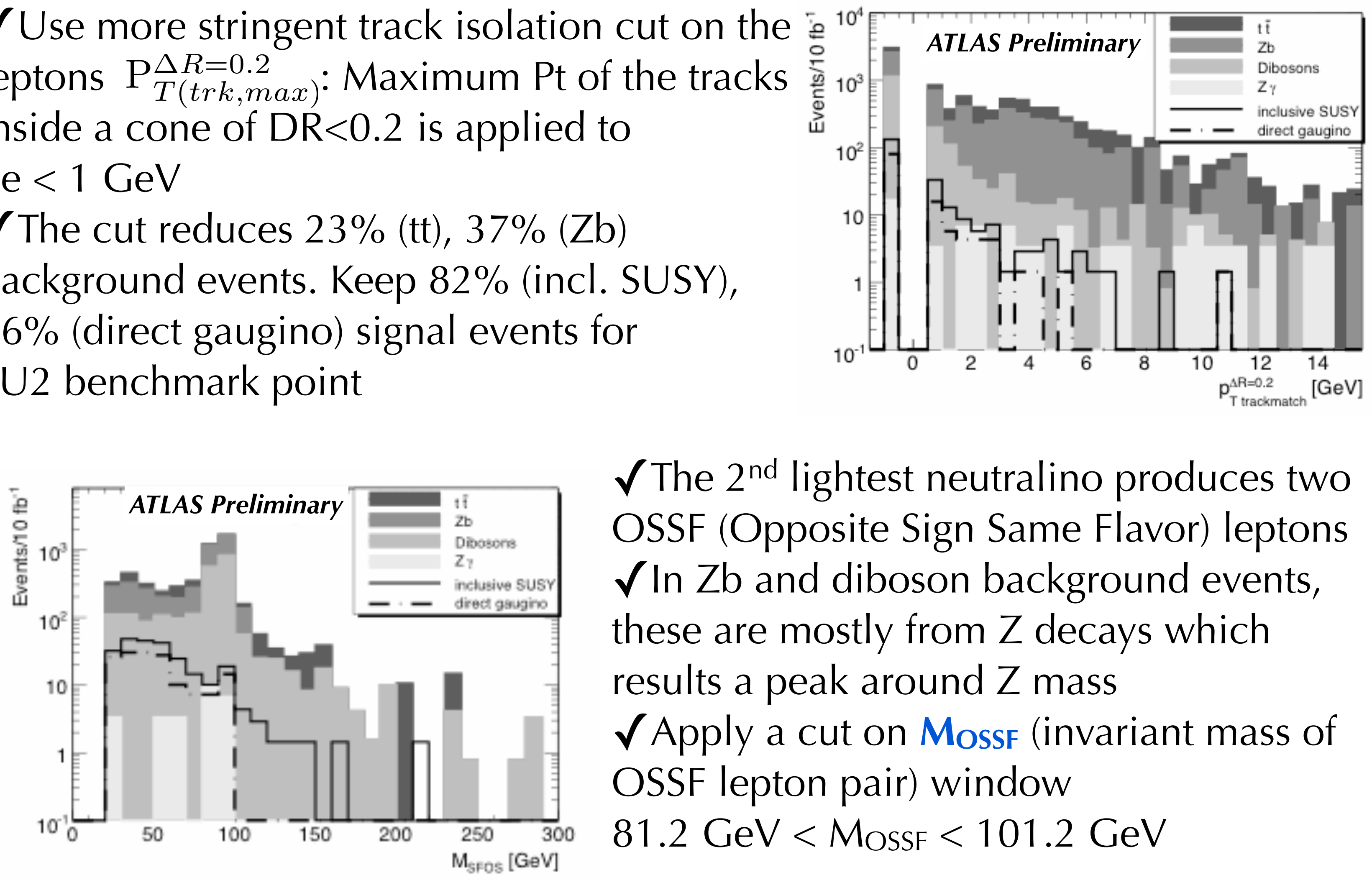

$\checkmark$ The $2^{\text {nd }}$ lightest neutralino produces two OSSF (Opposite Sign Same Flavor) leptons $\checkmark$ In $\mathrm{Zb}$ and diboson background events, these are mostly from $Z$ decays which results a peak around $Z$ mass $\checkmark$ Apply a cut on Mossf (invariant mass of OSSF lepton pair) window

81.2 GeV < M MSSF $<101.2 \mathrm{GeV}$ 


\section{Summary of Event Selection}

$\checkmark$ Minimum 1 OSSF lepton pair (e+e-, mu+mu-)

$\checkmark$ number of lepton $>=3$

$\checkmark \mathrm{P}_{T(\text { trk } m \text { max })}^{\Delta R=0.2}<2 \mathrm{GeV}$ (electrons) $<1 \mathrm{GeV}$ (muons)

$\checkmark$ Veto events which which falls within $81.2 \mathrm{GeV}<M_{\mathrm{OSSF}}<102.2 \mathrm{GeV}$ $\checkmark$ PtMiss $>30 \mathrm{GeV}$

$\checkmark$ Optional jet veto cut :

- Reject events containing jets with $\mathrm{Pt}>20 \mathrm{GeV}$

- Useful where the direct gaugino production dominates in the event rate

-ttbar background is suppressed with this cut
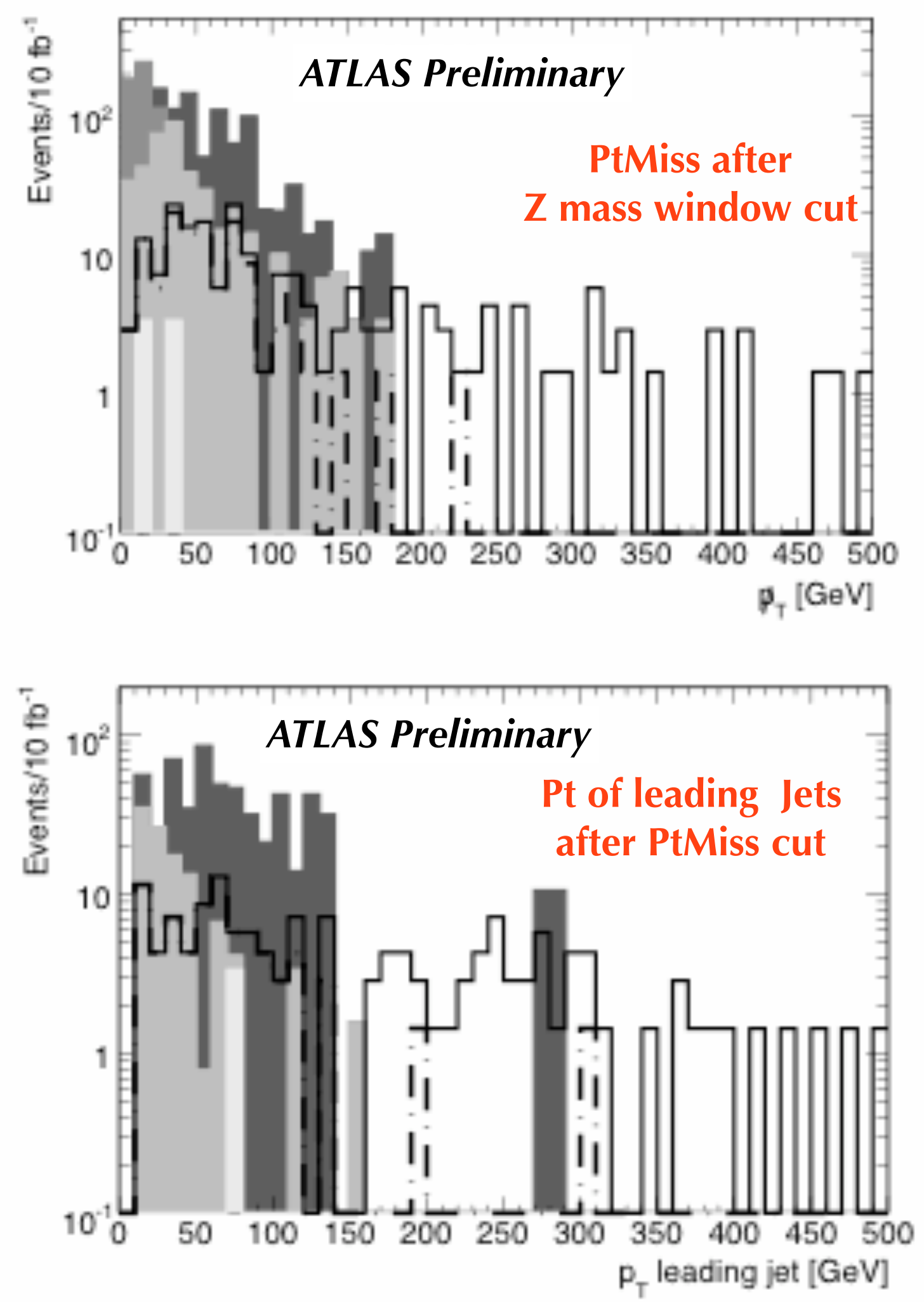


\section{Discovery potential}

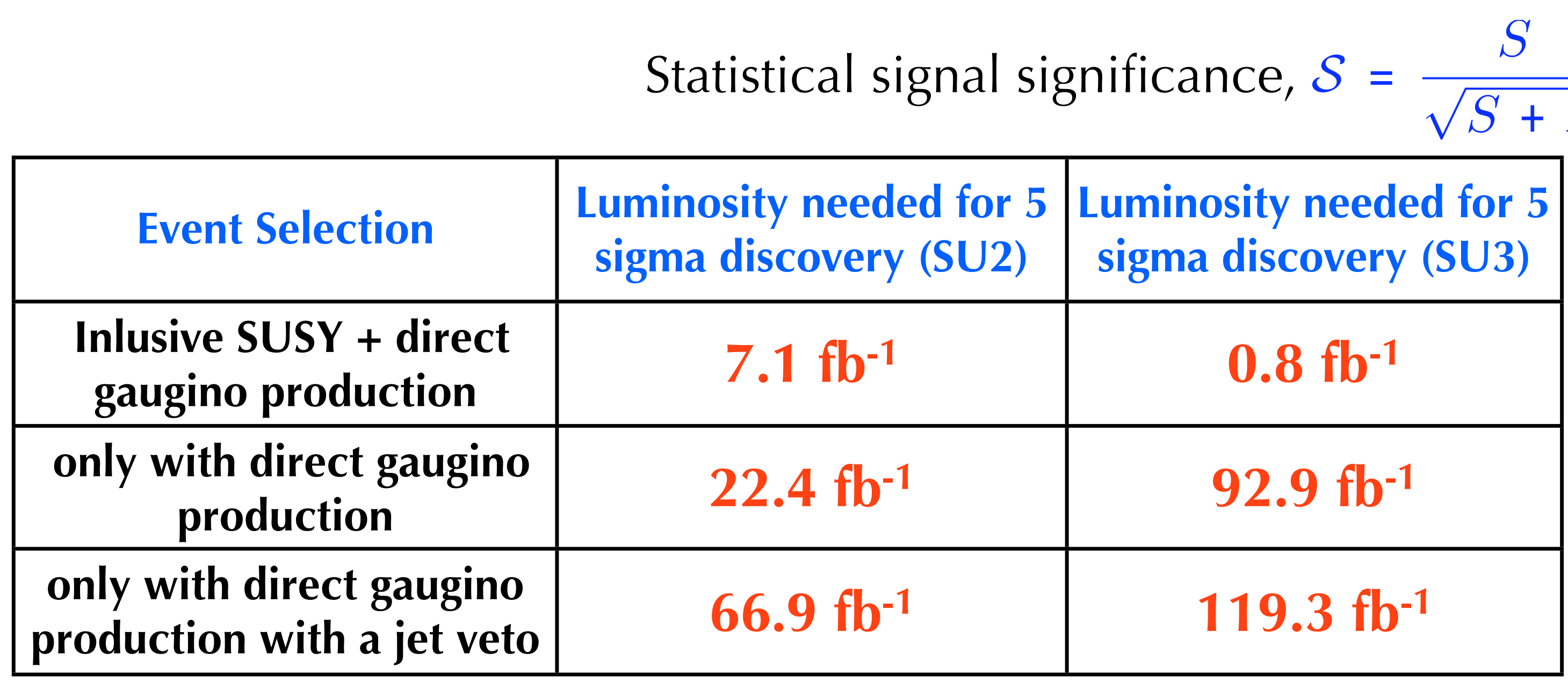

$\checkmark$ The Inclusive SUSY gives us a potential discovery

with a several $\mathrm{fb}^{-1}$ analysis for the two considered points

$\checkmark$ Benchmark point SU2 (focus point region) gives

promising result for measuring direct gaugino production

while more difficult for SU3 (bulk region) 


\section{Systematic uncertainties}

Source

Uncertainty

No jet veto With jet veto

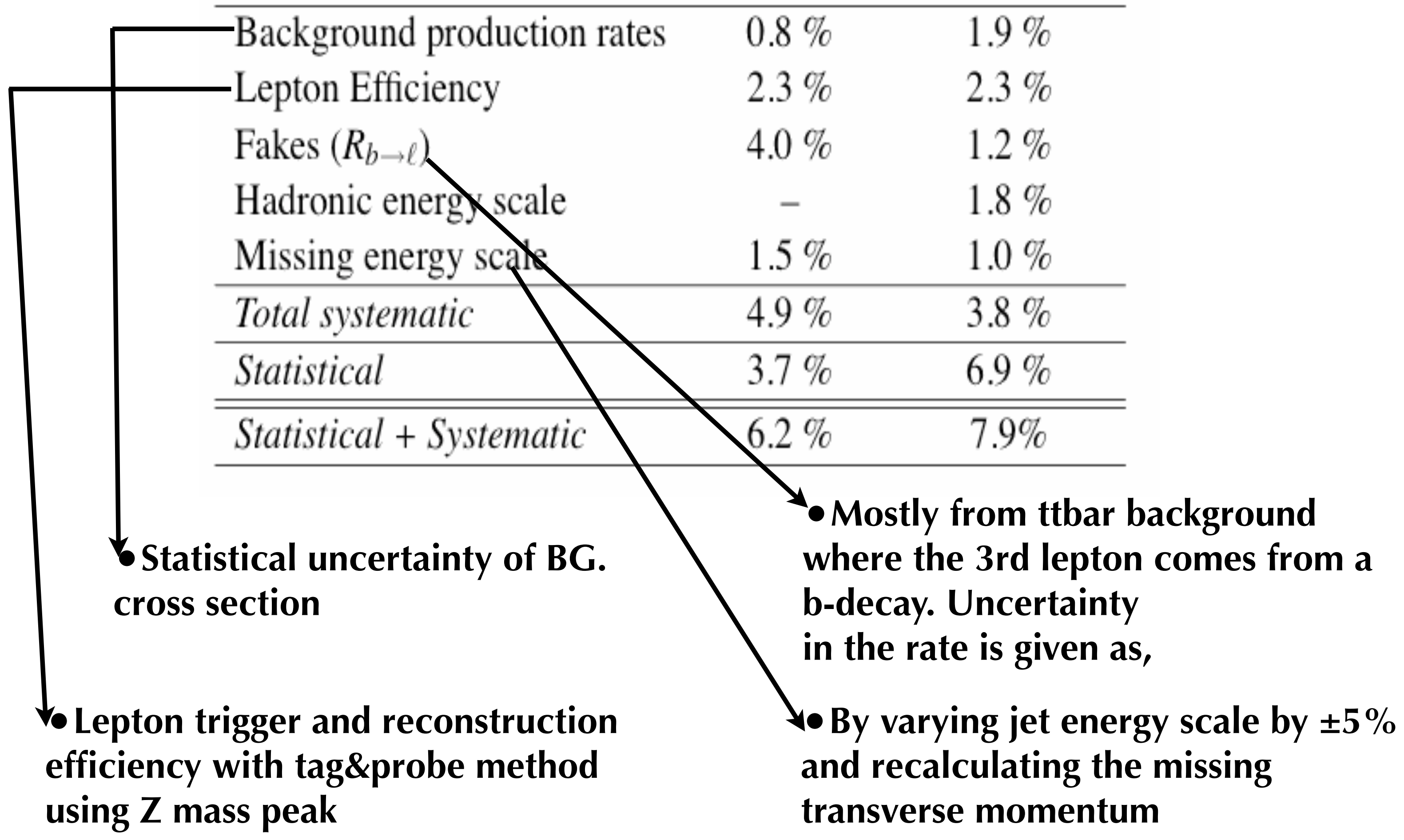




\section{Trigger Efficiency, Events with Pile-up}

\begin{tabular}{|c|c|c|c|c|c|c|c|c|c|}
\hline $\begin{array}{l}\text { Selection } \\
\text { stage }\end{array}$ & \multicolumn{3}{|c|}{ SU2 $\chi$} & \multicolumn{3}{|c|}{ SU3 $\chi$} & \multicolumn{3}{|c|}{ SU3 incl. } \\
\hline OSSF pair & $41 \%$ & $54 \%$ & $89 \%$ & $42 \%$ & $54 \%$ & $92 \%$ & $51 \%$ & $51 \%$ & $94 \%$ \\
\hline $\mathrm{OSSF}+3^{\mathrm{rd}} \ell$ & $58 \%$ & $67 \%$ & $93 \%$ & $59 \%$ & $63 \%$ & $95 \%$ & $66 \%$ & $68 \%$ & $98 \%$ \\
\hline after all cuts & $57 \%$ & $66 \%$ & $92 \%$ & $58 \%$ & $57 \%$ & $94 \%$ & $66 \%$ & $64 \%$ & $97 \%$ \\
\hline
\end{tabular}

[ after the event selection cuts, lepton triggers yields a very high efficiency ]
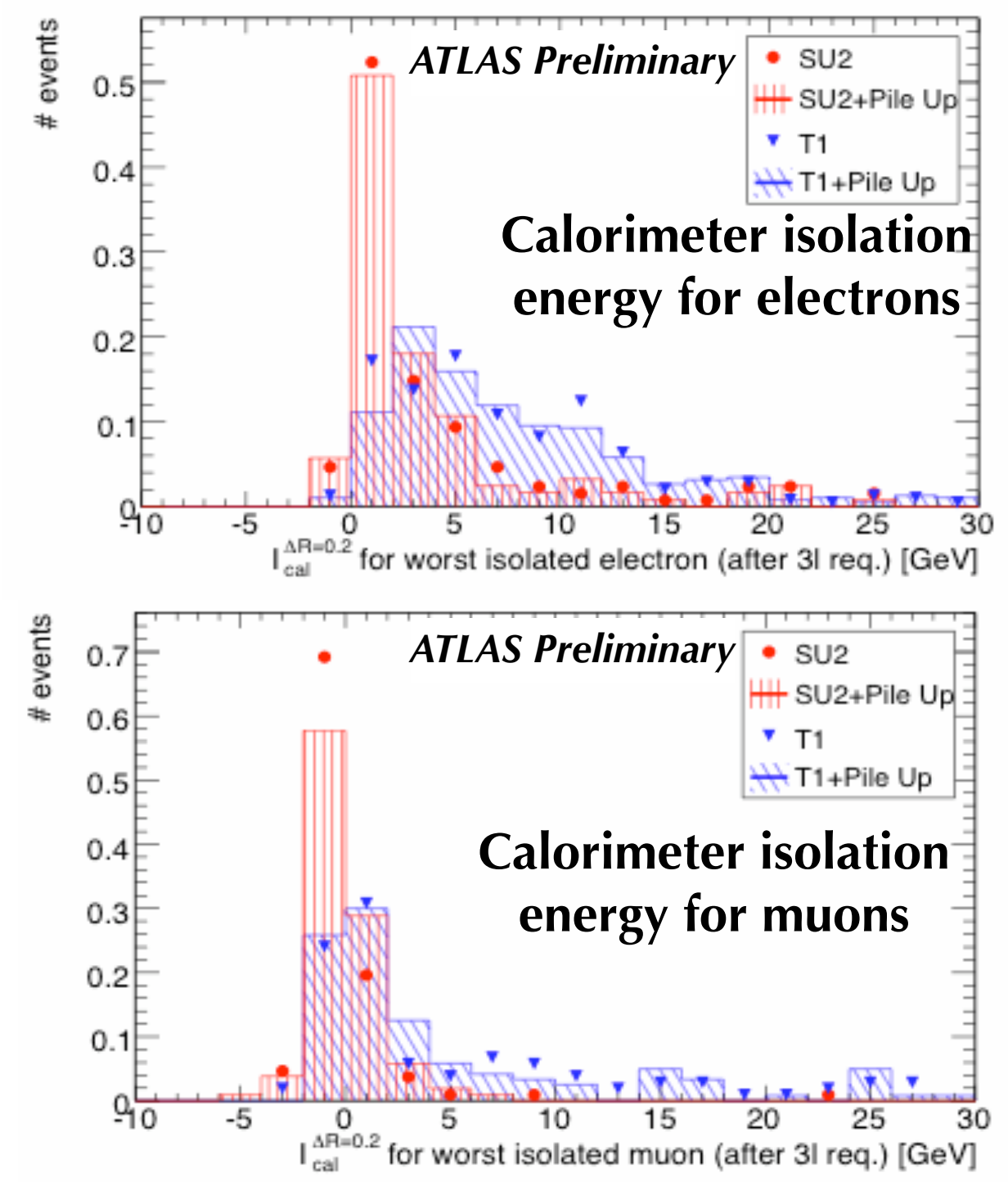

\begin{tabular}{|c|c|c|}
\hline Isolation & $e$ eff. (\%) & $\mu$ eff. (\%) \\
\hline \hline no isolation & $64.0 \pm 0.7(-1.3)$ & $74.1 \pm 0.6(-1.1)$ \\
\hline$E_{\text {cal }}^{\Delta R=0.2}<10 \mathrm{GeV}$ & $61.9 \pm 0.5(-1.7)$ & $74.0 \pm 0.4(-0.8)$ \\
\hline$p_{T \text { track,max }}^{\Delta R=}(\ell)<2 / 1 \mathrm{GeV}$ for $e / \mu$ & $57.6 \pm 0.5(-2.6)$ & $67.0 \pm 0.5(-3.5)$ \\
\hline
\end{tabular}

$\checkmark$ Loss of signal efficiency because of pile-up events in SU2 is $\sim 1 \%$ for calorimeter isolation and $\sim 3 \%$ for track isolation in LHC running at $10^{-33} \mathrm{~cm}^{-2} \mathrm{~s}^{-1}$ luminosity 


\section{Summary}

$\checkmark$ As, LHC is getting closer to begin data taking, ATLAS is preparing and exploring all aspects of SUSY for a discovery

$\checkmark$ Here, we presented the discovery potential of three lepton signature, missing transverse momentum and with a little jet activity in the final state

$\checkmark$ We consider several benchmark points within the mSUGRA parameter space, where as, focused our search to SU2 point. This point is specially chosen because of the heavy masses of the coloured sparticles which makes direct chargino, neutralino pair production to be the dominant process

$\checkmark$ Here, we found a discovery is possible in several $\mathrm{fb}^{-1}$ of $\mathrm{LHC}$ data for an inclusive signature without a jet veto, whereas, we need several tens of $\mathrm{fb}^{-1}$ data to do a discovery with a jet veto analysis

$\checkmark$ Methods to obtain background events using real data is underway $\checkmark$ More work on lepton efficiencies and fake rate estimation $\checkmark$ These will improve the overall performance of the analysis 


\section{BACKUP}




\section{Discovery potential in SU2 Vs other points}

\begin{tabular}{|c|r|r|r|r|r|r|r|r|}
\hline Kinematic Cut & No Cuts & $N_{L}>=2$ & \multicolumn{1}{c|}{ OSSF } & $N_{L}>=3$ & TrackIsol & $m_{\ell \ell}$ & $E_{T}$ & JetVeto \\
\hline Sample & & & & & & & & \\
\hline \hline SU2 gauginos & 64037 & 1647 & 1108 & 178 & 153 & 120 & 95 & 29 \\
\hline SU2 other & 7081 & 776 & 353 & 127 & 95 & 85 & 82 & 0 \\
\hline$t \bar{t}$ & 4406579 & 234656 & 104187 & 2812 & 634 & 507 & 476 & 42 \\
\hline$Z Z$ & 38202 & 10413 & 9984 & 580 & 476 & 57 & 13 & 6 \\
\hline$Z W$ & 156720 & 17224 & 14476 & 1910 & 1682 & 322 & 218 & 154 \\
\hline$W W$ & 400217 & 22708 & 10687 & 25 & 8 & 8 & 8 & 8 \\
\hline$Z \gamma$ & 32832 & 7184 & 6970 & 91 & 27 & 7 & 3 & 0 \\
\hline$Z b$ & 1591157 & 573601 & 559237 & 6523 & 2409 & 386 & 0 & 0 \\
\hline \hline inclusive SUSY $\mathscr{S}$ & & 2.60 & 1.74 & 2.76 & 3.36 & 5.31 & $\mathbf{5 . 9 4}$ & $\mathbf{1 . 8 7}$ \\
\hline direct gaugino $\mathscr{S}$ & & 1.77 & 1.32 & 1.61 & 2.09 & 3.20 & $\mathbf{3 . 3 4}$ & $\mathbf{1 . 8 7}$ \\
\hline
\end{tabular}

\begin{tabular}{|l|c|c|c|c|c|c|c|c|c|}
\hline & SU1 & SU2 & SU3 & SU4 & SU8 & SU2 & SU3 & SU2+JV & SU3+JV \\
\hline \hline $\mathscr{S}, 10 \mathrm{fb}^{-1}$ & 7.7 & 5.9 & 17.2 & 69.3 & 1.9 & 3.3 & 1.6 & 1.9 & 1.4 \\
$\int \mathrm{d} t \mathscr{L}$ for $5 \#$ & 4.2 & 7.1 & 0.8 & 0.1 & 70.5 & 22.4 & 92.9 & 66.9 & 119.3 \\
\hline
\end{tabular}




\section{Flavor subtracted Invariant mass}
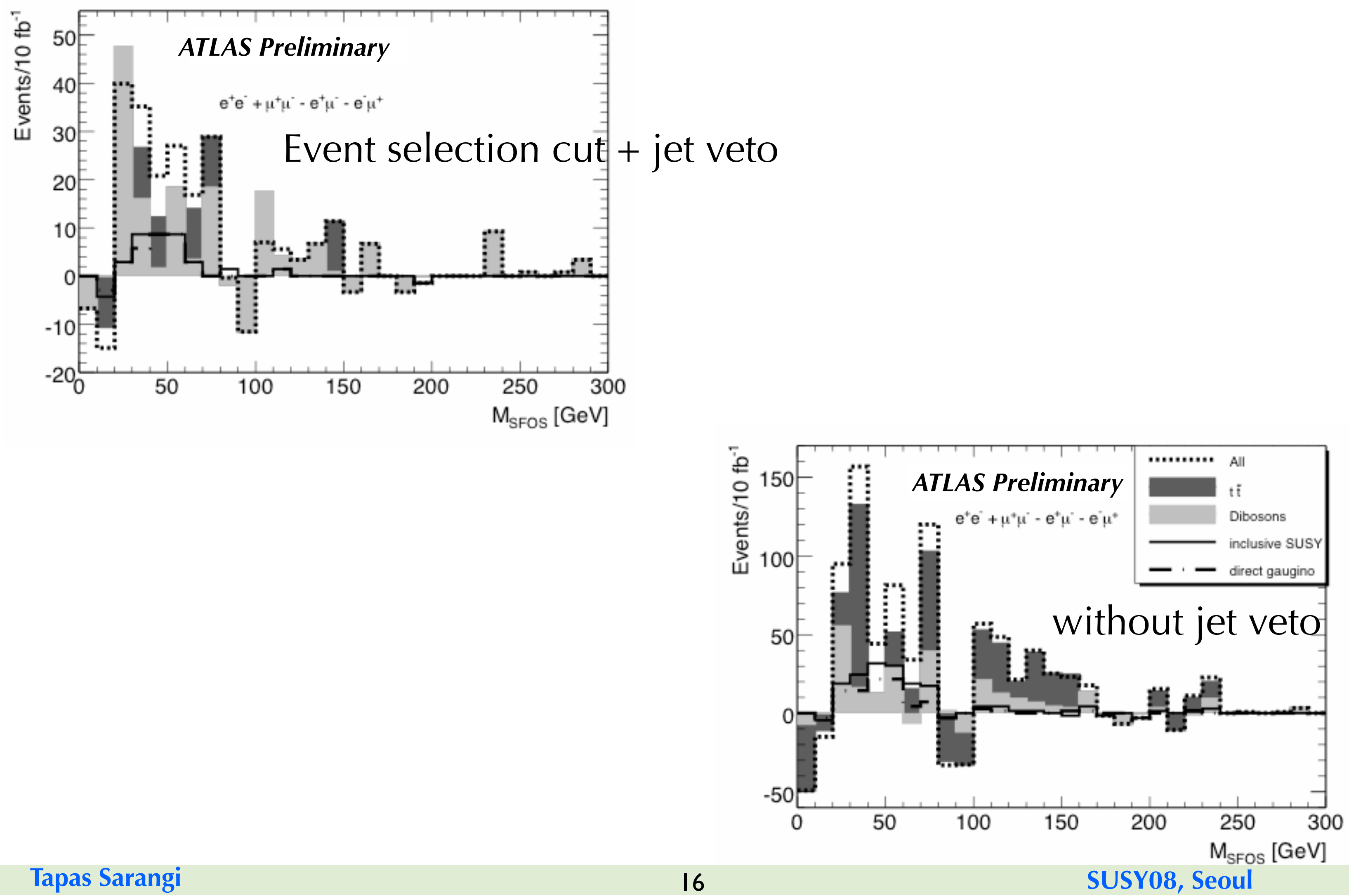


\section{LHC and ATLAS}

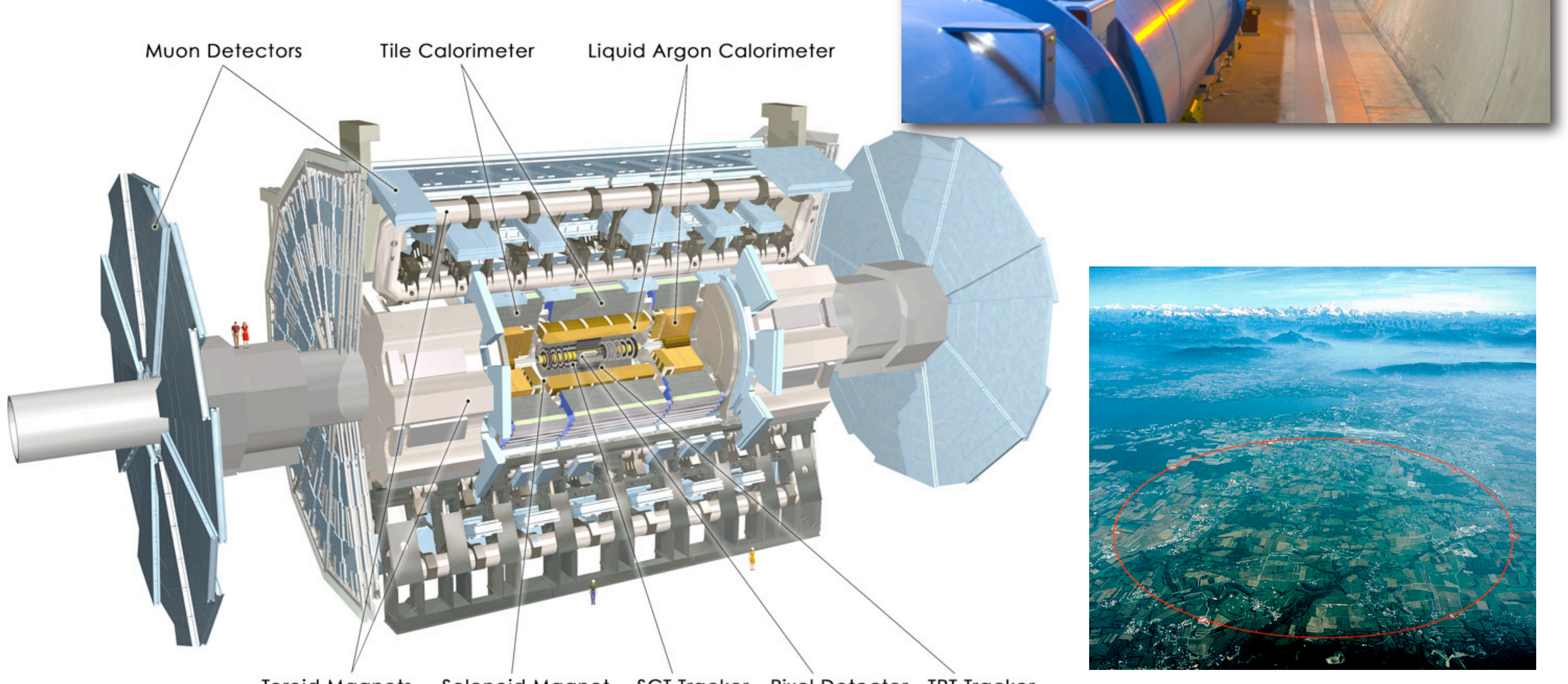

